\title{
X-tra X: An escape to autoimmunity
}

\author{
Gregory F. Wu
}

Department of Neurology, Department of Pathology and Immunology, Washington University in St. Louis School of Medicine, St. Louis, Missouri, USA

\begin{abstract}
Identifying the factors driving disease disparities between males and females with multiple sclerosis (MS) holds great promise for deciphering immunopathogenic disease mechanisms. In this issue of $J C l$, Itoh et al. explore the basis for sexual dimorphism in autoimmunity, specifically in MS. Using the experimental autoimmune encephalomyelitis (EAE) model of MS, which recapitulates $\mathrm{CD}^{+} \mathrm{T}$ cell-dependent disease, the authors examined the contribution of $K d m 6 a$, a histone demethylase gene known to escape $\mathrm{X}$ inactivation. Conditional knockout in $\mathrm{CD}^{+}{ }^{+} \mathrm{T}$ cells revealed $\mathrm{Kdm6a}$ involvement with a collection of immunologic processes having the potential to skew immunity toward inflammatory responses. This study concisely shows the value of $X$ chromosome gene expression in $T$ cell regulation of autoimmunity and the relevance of $K d m 6 a$ in the pathogenesis of EAE as a model of MS.
\end{abstract}

\section{Basis for sexual dimorphism in multiple sclerosis}

Like many other autoimmune diseases, multiple sclerosis (MS) affects females in a greater proportion compared with males (1). In addition to a three-fold higher incidence in females, onset and severity of disease may differ as well (2). Exploring disease heterogeneity between patients, while vexing, may offer insights into fundamental pathways involved in the disease state common to all patients.

Much of the basis for sexual dimorphism in MS has been attributed to hormonal disparities, a hypothesis that has led to clinical trials involving estrogen or testosterone treatment (3). Yet a more fundamental basis for the discrepancy in MS prevalence among females may lie with the $\mathrm{X}$ chromosome itself. Inactivation, an epigenetic regulatory process, limits $\mathrm{X}$ chromosome gene expression in females, leaving only one $\mathrm{X}$-linked gene copy active in any given cell. However, some genes escape suppression and are expressed in greater abundance. A major challenge is understanding the impact of gene inactivation escape and how dosage effects of certain genes participate in the genesis of autoimmune diseases.

\section{Contribution of Kdm6a}

$\mathrm{Kdm} 6 \mathrm{a}$ is a histone demethylase known to escape $\mathrm{X}$ inactivation. In this issue, Itoh and colleagues report on their use of the experimental autoimmune encephalomyelitis (EAE) mouse model of MS to explore the contribution of Kdm6a (4). They observed that $K d m 6 a$ expression was markedly elevated in $\mathrm{CD} 4^{+} \mathrm{T}$ cells from females compared with males in both naive mice and healthy human subjects. Itoh et al. then employed a conditional knockout system to determine the effects of $\mathrm{Kdm} 6 a$ loss specifically in $\mathrm{CD} 4{ }^{+}$ $\mathrm{T}$ cells. Conditional knockout mice had a greater abundance of splenic naive $\mathrm{CD} 4{ }^{+}$ $\mathrm{T}$ cells, with a concomitant decrease in $\mathrm{CD} 44^{\text {hi }} \mathrm{CD} 62 \mathrm{~L}^{\text {lo }} \mathrm{CD} 4^{+} \mathrm{T}$ cells compared with controls. This is a provocative obser-

Related Article: p. 3852

Conflict of interest: GFW has served on advisory boards for Novartis and EMD Serono, received compensation for speaking on behalf of Sanofi, and is a consultant with the US Department of Justice.

Copyright: @ 2019, American Society for Clinical Investigation.

Reference information: / Clin Invest. 2019;129(9):3536-3538. https://doi.org/10.1172/JCI130312.

vation, given that circulating memory $\mathrm{T}$ cells have been repeatedly observed in a higher proportion in MS patients than in healthy individuals (5). Hence, the development of MS, which is usually diagnosed in adulthood, may stem from an overabundance of Kdm6a that over years favors a gradual accumulation of activated and memory $\mathrm{T}$ cells.

Itoh and colleagues then determined whether a loss of $K d m 6 a$ in $\mathrm{CD} 4^{+} \mathrm{T}$ cells affected neuroinflammation in EAE. Using the traditional EAE model, which involves active immunization of C57BL/6 mice with $\mathrm{MOG}_{35-55}$, the authors found that loss of $K d m 6 a$ in $\mathrm{CD}^{+} \mathrm{T}$ cells was associated with markedly lower disease scores. Further, $\mathrm{CD} 4^{+} \mathrm{T}$ cells from conditional knockout mice stimulated with $\mathrm{MOG}_{35-55}$ ex vivo secreted less IL-2 compared with T cells from WT controls, while production of IL-5 was elevated. These results support the notion that $\mathrm{CD} 4^{+} \mathrm{T}$ cells depend on $\mathrm{Kdm} 6 \mathrm{a}$ to exhibit encephalitogenicity. Canonical pathway analysis of gene expression in Kdm6a knockout $\mathrm{CD} 4^{+} \mathrm{T}$ cells revealed upregulation of the Th2 pathway, among others. Downregulated pathways included neuroinflammation signaling, TLR signaling, and IL-17 signaling.

These findings fit nicely with the concept that genes escaping $\mathrm{X}$ inactivation promote autoimmunity (6). Interestingly, a previous study on Kdm6a in thymic development (7) showed that Kdm6a promotes expression of S1PR1, which is a target of the FDA-approved disease-modifying therapies for MS fingolimod and siponimod. Minor sex-dependent differences in side effects and disability profiles have been reported in patients treated with fingolimod $(8,9)$; however, sex-specific differences in efficacy in response to S1PR1related modulators that are dependent on $\mathrm{T}$ cell function have not been reported to date. Still, the effects of $K d m 6 a$ in T cells could be the result of a combination of effects across the genome that influence several genes, such as S1PR1, resulting in an eventual breach of the threshold to autoimmunity (Figure 1). 

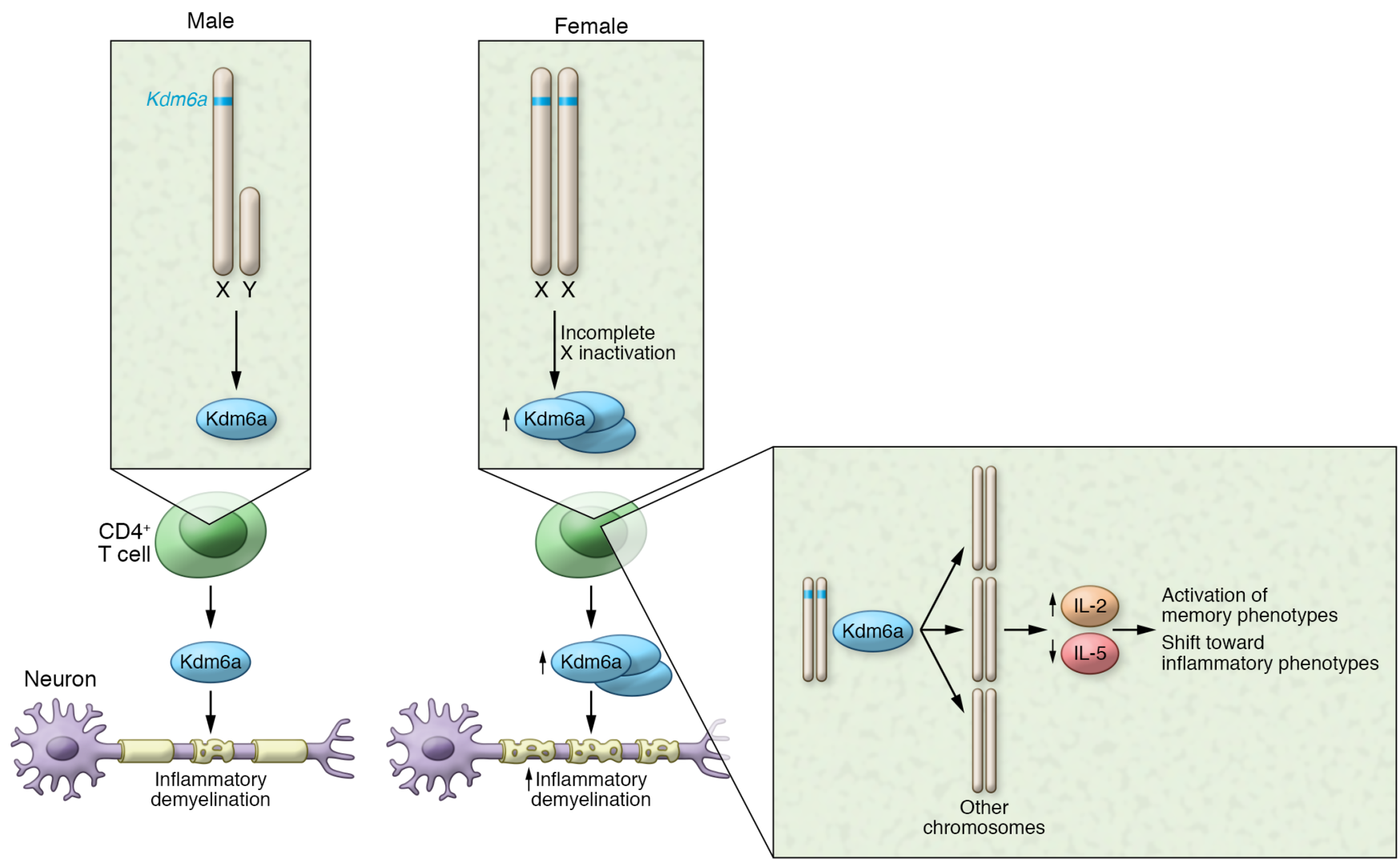

Figure 1. X-linked histone demethylase Kdm6a influences development of autoimmunity. Kdm6a is present on the $\mathrm{X}$ chromosome and is expressed at higher levels in CD4+ $T$ cells from females than those from males. Kdm6a alters expression of immune response genes, resulting in expression patterns that are linked to neuroinflammation and demyelination. In both male and female mice, deletion of $K d m 6 a$ in CD4 ${ }^{+} \mathrm{T}$ cells ameliorated neuropathology during EAE.

\section{Conclusions and future directions}

The identification of $K d m 6 a$ in $\mathrm{CD}^{+} \mathrm{T}$ cell autoimmunity may provide insight into more generalized mechanisms of MS susceptibility in both sexes as well as autoimmunity in general. Aside from $\mathrm{Kdm} 6 \mathrm{a}$ knockout analysis in $\mathrm{CD}^{+}$cells, downstream effects via multiple genes were not fully explored by Itoh et al. One approach would be to systematically characterize Kdm6a demethylation targets that are also implicated in MS susceptibility, such as gene candidates associated with vitamin D levels (10).

One intriguing finding of this study involves the potential effect of $K d m 6 a \mathrm{X}$ inactivation in $\mathrm{CD}^{+} \mathrm{T}$ cells. Because $\mathrm{T}$ cell progenitors in the thymus express $C d 4$ during development, the use of Cd4-Cre by Itoh and colleagues also results in loss of $\mathrm{Kdm} 6 \mathrm{a}$ in $\mathrm{CD}^{+}{ }^{+} \mathrm{T}$ cells. Since there is accumulating evidence for a suppressive function for $\mathrm{CD}^{+} \mathrm{T}$ cells in some models of EAE and in MS, it would be important to determine the influence of $K d m \sigma a$ in $\mathrm{CD}^{+} \mathrm{T}$ cell development and/or effector functions relevant to autoimmunity and whether or not $\mathrm{Kdm} 6 \mathrm{a}$ expression in $\mathrm{CD}^{+} \mathrm{T}$ cells contributes during EAE (11). Reconciling the proinflammatory nature of $K d m 6 a$ in $\mathrm{CD}^{+}$ $\mathrm{T}$ cells with any potential gene modulation favoring antiinflammatory features of $\mathrm{CD} 8^{+}$ $\mathrm{T}$ cells will therefore be highly interesting and justifies further study.

Perhaps of equal interest is how $K d m 6 a$ uniquely fosters differences between female and male $\mathrm{CD} 4^{+} \mathrm{T}$ cells, while other genes known to escape $\mathrm{X}$ inactivation are not immediately implicated. Though Itoh et al. did not directly investigate other genes in their study, they note that escape from $\mathrm{X}$ inactivation depends on factors such as tissue type, cell type, and hormonal status. Hence, the complexity of MS pathogenesis would naturally be compounded by gene regulation in different tissues at different times. Overall, the experiments reported by Itoh et al. uncover potential mechanisms involving $\mathrm{X}$ inactivation in $\mathrm{T}$ cells during MS, and devising any therapeutic approach revolving around $K d m 6 a$ targeting should consider biological context.

Broadly speaking, the work by Itoh et al. informs the field on the pathogenesis of MS in several ways. First, the basis for sexual dimorphism in MS unmistakably extends beyond hormones alone. Implication with $\mathrm{X}$ inactivation escape adds to the tremendous complexity of MS pathogenesis, perhaps tempering expectations regarding hormone-based trials in MS. Second, it emphasizes the paradigm of $\mathrm{T}$ cell-mediated inflammatory responses that are integral to MS susceptibility. While the EAE data is derived from the a priori dependence of $\mathrm{CD} 4^{+} \mathrm{T}$ cells, the human data presented certainly support the notion that $\mathrm{CD} 4^{+} \mathrm{T}$ cells are a primary contributor to the pathogenesis of MS. Finally, mechanisms involved in $\mathrm{T}$ cell autoreactivity in males can be uncovered by exploring unique features of autoimmunity in females. It is important to recognize that Itoh et al. observed that loss of $K d m 6 a$ in male $\mathrm{CD}^{+} \mathrm{T}$ cells also was associated 
with amelioration of EAE. Consequently, Kdm6a demethylase activity shows effects that extend beyond the $\mathrm{X}$ inactivation that occurs in females. Specifically, Kdm6a contributes to $\mathrm{CD}^{+} \mathrm{T}$ cell encephalitogenic behavior in males. Hence, identification of Kdm6a activity may allow for assessment of gene targets, independent of sex. Together, these findings leverage the heterogeneity of MS nicely and set the stage for further examination of the $\mathrm{T}$ cell foundation for sex differences in MS susceptibility and autoimmunity in general.

\section{Acknowledgments}

The author thanks Brian T. Edelson, Rajendra S. Apte, and Alfred H.J. Kim for critical reading of the manuscript. Funding support for the author comes from National Institute of Neurological Disorders and Stroke R01 NS106289. GFW has received research support from Biogen,
EMD Serono, The Doris Duke Charitable Foundation, Genentech, the NIH, and the National Multiple Sclerosis Society.

Address correspondence to: Gregory F. Wu, Box 8111, 660 S. Euclid Avenue, St. Louis, Missouri 63110, USA. Phone: 314.362.3293;

Email:wug@neuro.wustl.edu.

1. McCombe PA, Greer JM, Mackay IR. Sexual dimorphism in autoimmune disease. Curr Mol Med. 2009;9(9):1058-1079.

2. Bove R, Chitnis T. Sexual disparities in the incidence and course of MS. Clin Immunol. 2013;149(2):201-210.

3. Collongues N, Patte-Mensah C, De Seze J, Mensah-Nyagan AG, Derfuss T. Testosterone and estrogen in multiple sclerosis: from pathophysiology to therapeutics. Expert Rev Neurother. 2018;18(6):515-522.

4. Itoh Y, et al. The X-linked histone demethylase Kdm6 $\mathrm{a}$ in $\mathrm{CD}^{+}{ }^{+} \mathrm{T}$ lymphocytes modulates autoimmunity. JClin Invest. 2019;129(9):3852-3863.

5 . Bose T. Role of immunological memory cells as a therapeutic target in multiple sclerosis. Brain Sci. 2017;7(11):E148.

6. Brooks WH. X chromosome inactivation and autoimmunity. Clin Rev Allergy Immunol. 2010;39(1):20-29.

7. Manna S, et al. Histone H3 Lysine 27 demethylases Jmjd3 and Utx are required for T-cell differentiation. Nat Commun. 2015;6:8152.

8. Manni A, et al. Gender differences in safety issues during Fingolimod therapy: Evidence from a real-life Relapsing Multiple Sclerosis cohort. Brain Behav. 2017;7(10):e00804

9. Devonshire V, et al. Relapse and disability outcomes in patients with multiple sclerosis treated with fingolimod: subgroup analyses of the double-blind, randomised, placebocontrolled FREEDOMS study. Lancet Neurol. 2015;11(5):420-428.

10. Manousaki D, et al. Low-frequency synonymous coding variation in CYP2R1 has large effects on vitamin D levels and risk of multiple sclerosis. Am J Hum Genet. 2017;101(2):227-238.

11. Sinha S, Boyden AW, Itani FR, Crawford MP, Karandikar NJ. CD8(+) T-cells as immune regulators of multiple sclerosis. Front Immunol. 2015;6:619. 